\title{
Strong starlight suppression sufficient to enable direct detection of exoplanets in the habitable zone ${ }^{\star}$
}

\author{
S. R. Martin ${ }^{1}$ and A. J. Booth ${ }^{2}$ \\ 1 Jet Propulsion Laboratory, California Institute of Technology, 4800 Oak Grove Drive, Pasadena, California 91109, USA \\ e-mail: stefan.r.martin@jpl.nasa.gov \\ 2 Sigma Space Corp., 4801 Forbes Boulevard, Lanham, MD 20706, USA
}

Received 26 January 2010 / Accepted 4 February 2010

ABSTRACT

\begin{abstract}
Direct detection of photons from exoplanets in the habitable zone around nearby stars is challenging because of the much higher photon flux and close angular proximity of the star. At mid-infrared wavelengths, around $10 \mu \mathrm{m}$, the flux contrast ratio between a sun-like star and an earth-size planet is several million to one, favorable compared to the visible range, while the angular separation of the bodies is less than $1 \mu$ radian.

The wavelength range between 7 and $20 \mu \mathrm{m}$ is worthy of study because it can yield information on the planetary atmospheric composition, particularly as it contains absorption lines for $\mathrm{CO}_{2}$, ozone and water, which together can be considered a biomarker under some conditions. To achieve observations of the spectrum, strong and stable suppression of the excess starlight is required along with suppression of the local and exo-Zodiacal light, which also have much higher fluxes than the exoplanet signal.

Here we show in the laboratory with nulling interferometry, the suppression of artificial starlight by almost eight orders of magnitude, which is sufficient to detect a planet some three million times fainter than the star. The results show that a combination of starlight suppression techniques enables the detection of medium-sized planets in the habitable zone around nearby stars. Large space telescopes planned for future exoplanet studies will employ these techniques and one additional method, which brings earth-size planets within reach to obtain compelling data on the atmospheres of nearby exoplanets.
\end{abstract}

Key words. techniques: interferometric - planets and satellites: detection

\section{Introduction}

In the technique of nulling interferometry (Bracewell 1978), radiation from the target object is collected through two separate apertures and combined after inverting the electric field of one of the inputs. The resultant Young's fringes (seen on the sky) are destructive along the line of sight, so that radiation from a pointlike target is extinguished. Light from objects at angles $\lambda / 2 B$, $3 \lambda / 2 B$, etc. (where $\lambda$ is the wavelength and $B$ is the interferometric baseline, that is the distance between the aperture centers) is constructively interfered and may be detected. Additional nulling aperture pairs may be added to enable phase modulation of the field of view, for example on ground-based telescopes (Colavita et al. 2006) in order to reject atmospheric disturbances to the amplitude and phase of the incoming beams pairs. For a space telescope, one or two dimensional arrays (Mennesson \& Mariotti 1997) using phase modulation in combination with rotation of the aperture array around the line of sight, would be used to reduce thermal background including local and exoZodiacal radiation and instrument disturbance effects to enable detection of faint exoplanets. The effect of the array rotation is to rotate the interference fringes on the sky, producing characteristic planet signals, which may be detected through matched filtering. Figure $1 \mathrm{~A}$ shows the two-dimensional on-sky fringe pattern for TPF-Emma (Martin et al. 2007). The star would be maintained in the central dark nulled region, and radiation from a planet located at the radius of the strong interference fringes

\footnotetext{
^ Formerly at the Jet Propulsion Laboratory.
}

would be modulated as the array rotates as shown in Fig. 1B, see for example, Defrere et al. (2009).

\section{Planet detection testbed}

The exoplanet signal detection process is illustrated in Fig. 2. Approximating the intensities in terms of orders of magnitude, top left, the star's intensity is normalized to unity and the local Zodiacal background light has an intensity of $10^{-4}$. The starlight would be nulled to the $10^{-5}$ level and then rotation of the interferometer array, averaging, matched filtering, and finally spectral fitting (Lay 2006) reduce the starlight to $10^{-8}$ of the original intensity. The planet detection testbed (Booth et al. 2008) (PDT) has been developed for NASA's terrestrial planet finder interferometer (TPF-I) program to test this method of exoplanet detection using mid-infrared nulling. In our laboratory experiment, performed on the PDT, the light from a bright monochromatic source (forming the star) is split into four identical beams and may be combined with four beams of light from a faint source (forming the planet). For the test outlined here, we did not use the planet source. The four beams are then combined in a four-beam nulling interferometer, which eliminates most of the starlight by nulling the beams in pairs. The outputs of the two nullers are then combined and a phase modulation is also applied, enabling homodyne detection of the signal via a lock-in amplifier. The testbed embodies the main systems required for a large infrared telescope array (Martin 2005) such as fringe tracking for phase-locking on the star and laser metrology for internal 

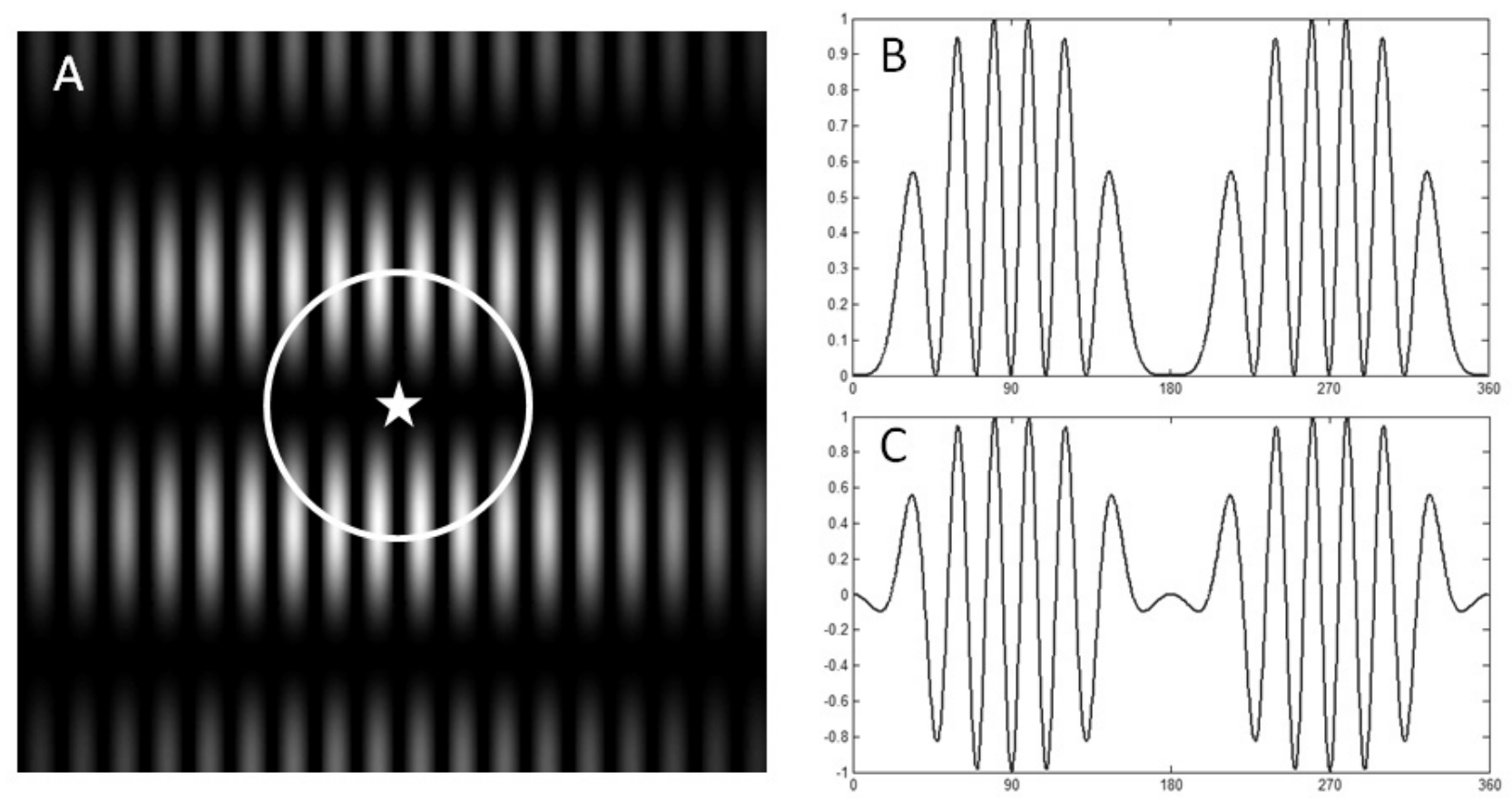

Fig. 1. A: Intensity map of the fringes on the sky. The star is at the center and the array rotation causes the planet to move along the circular path. B: Normalized intensity signal from planet as the array rotates through $360^{\circ}$. C: Planet template signal of unity amplitude produced by phase chopping. This signal was used as the matched filter to test the noise suppression.

pathlength and alignment control. Since the testbed's beam combination scheme is the same as that of TPF-Emma, it produces realistic signals which can be used to test starlight suppression and noise rejection. The output data can then be processed to test for the presence of planetary companions to the artificial star. Since, in this case, there are no companions, the test measures the noise level at the chosen radius, which was at the peak of the first constructive fringe on the nulling baseline.

On the right of Fig. 2, the test described here is illustrated, reproducing all but one of the exoplanet detection steps. The starlight will be nulled to the $10^{-5}$ level and then averaging and matched filtering (testing for specific planet signatures, which would be created by the rotation of the interferometer array) will reduce the starlight to at most $10^{-7}$ of the original intensity. This level therefore forms the baseline noise level. Since the testbed currently uses a monochromatic source for the star, the spectral fitting step shown on the left, which requires a broadband source and is used to reduce the instability noise (Lay 2004), cannot yet be executed.

We report here the result of an experiment made with the PDT showing strong suppression of the starlight so that the characteristic signal of a planet $\approx$ three million times fainter than the star would be detectable at a signal to noise ratio of 10 . At this suppression level, the ultimate goal of earth-sized exoplanet detection is not quite reached, but Neptune-sized planets (that are approximately three to four times larger) would be readily detectable in the habitable zone. Two planets in this size range (Kepler-4b and GJ1214b) were recently detected by transit methods (Borucki et al. 2010; Charbonneau et al. 2009). The expected star-to-planet contrast ratio would vary by a factor of $\approx 25$ across the spectral band (Des Marais et al. 2002), so a single number cannot cover all cases and a number of other factors would affect performance, for example, stellar diameter, zodiacal light and distance to the target system (Defrere et al. 2008). The figures used here therefore should be considered illustrative

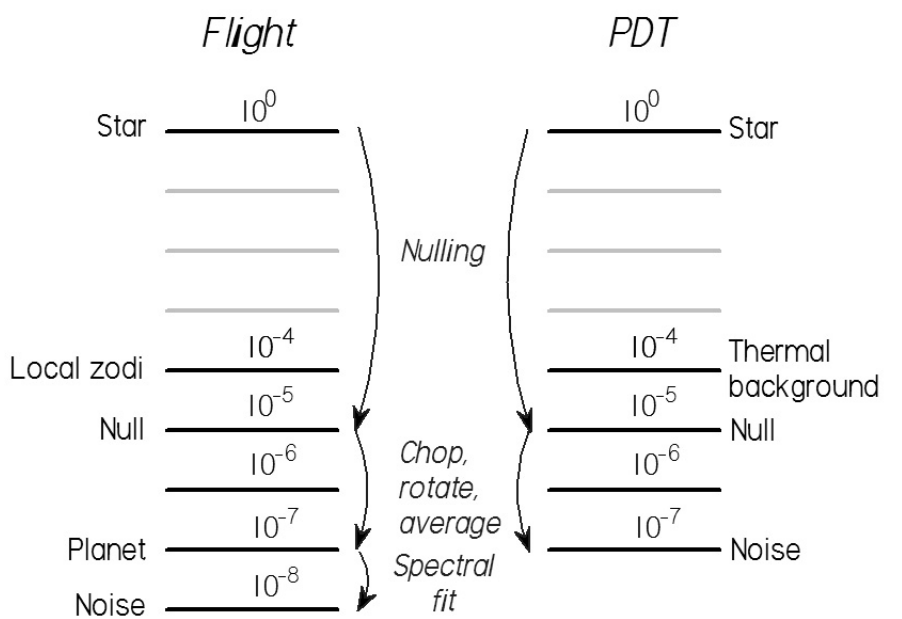

Fig. 2. Exoplanet detection process using a nulling interferometer.

of an observation made in a portion of that wavelength range. True earth-sized planet detection requires the addition of the spectral fitting technique to the methods outlined here: this is planned as the next phase for PDT.

Since the testbed operates in a normal laboratory environment rather than under a vacuum at cryogenic temperatures, some steps were taken to allow traceability of the testbed performance to flight. First, for the test discussed here, the null depth (the suppression of the starlight due to nulling) was limited to approximately $10^{-5}$ in order to emulate the suppression that would be used in space. Near the earth, the local zodiacal light and the exo-zodiacal light would total around $10^{-4}$ of the flux from the target star. Therefore there is little to be gained by suppressing the starlight by more than about five orders of magnitude. Secondly, the test was run over approximately three hours rather than the ten hours typically needed in space. To compensate for 
S. R. Martin and A. J. Booth: Strong starlight suppression sufficient to enable direct detection of exoplanets
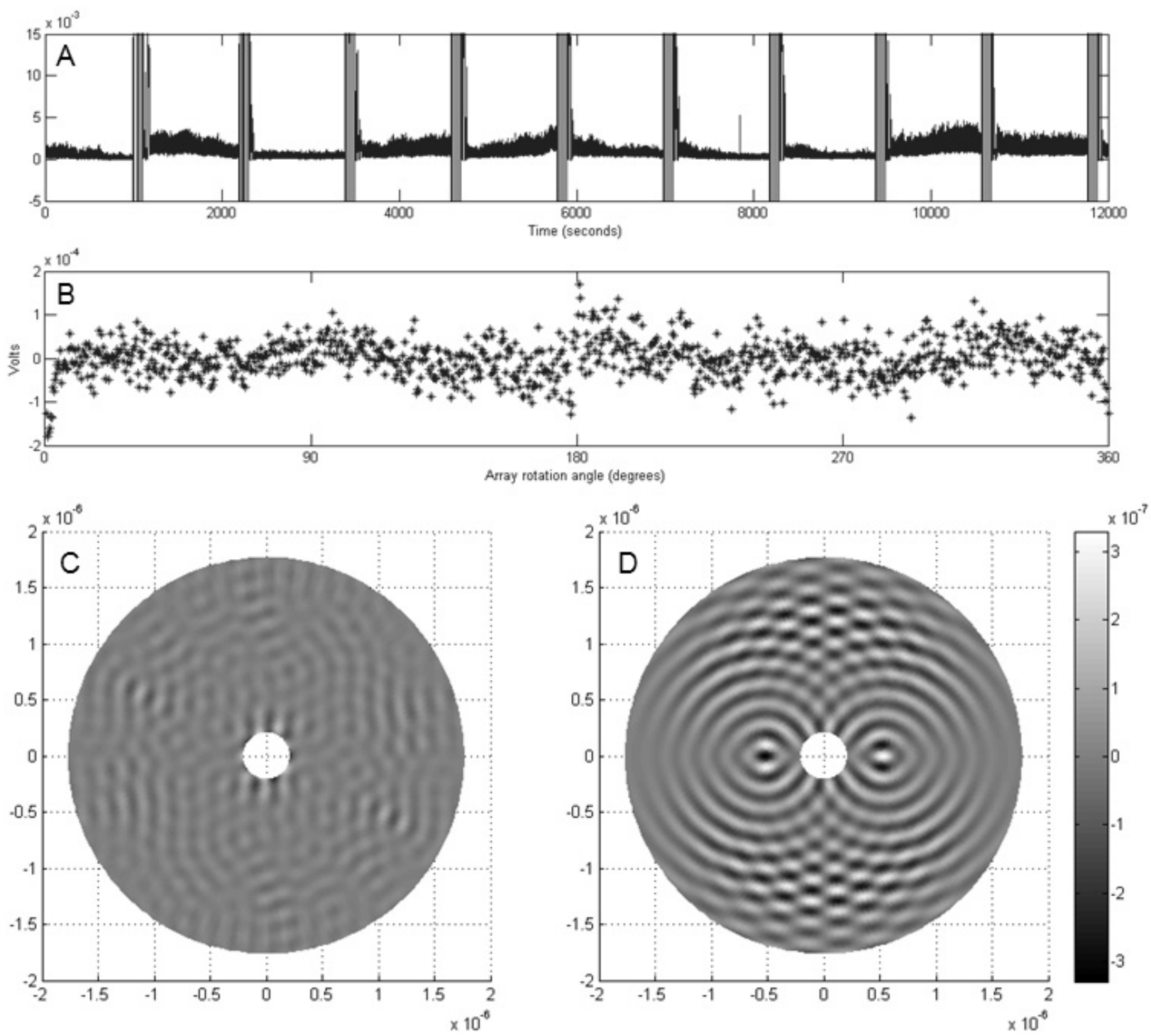

Fig. 3. A: Data from the detector. The periodic full scale excursions are the resets. B: Data with processing steps applied: phase chopping, averaging over five rotations and filtering. The vertical axis shows volts measured at the detector: the full flux from the star would produce $254 \mathrm{~V}$ on this scale. C: Central part of interferometer field of view with a $0.2 \mu$ radian region around the star excluded. The map shows the measured noise amplitude $(\Phi)$ in space for trace B. D: For comparison with C, at identical scales, a model of the signal from a planet at location $\left(0.53 \times 10^{-6}, 0\right)$, to the right of center. The planet intensity is $3.3 \times 10^{-7}$ of the star.

the resulting increased noise level, five array rotations were allowed in the analysis phase, rather than a single one. Thirdly, the testbed has nulling stability similar to that required in space, so that it produces a similar level of instability noise. Separate algebraic and numerical models were used to verify that this setup is a good analogue of the performance in space.

After nulling the two pairs of input beams, the nulled output beams were combined at a beamsplitter known as the crosscombiner and then sent to the detector. The cross-combiner executes a step-like motion (known as phase chopping), which causes the phase between the nuller outputs to alternate by one half wavelength, modulating the constructive areas of the fixed nulling fringe pattern. This modulated signal was then processed to test the starlight suppression. Figure $3 \mathrm{~A}$ shows the detector output measured in Volt. The total input starlight would measure $254 \mathrm{~V}$ on this scale. The horizontal axis of the figure represents time and extends over a nominal five rotations of the array, lasting a total of approximately three hours. Periodically, the testbed was automatically reset to counteract any drifting of the alignment; this results in the full scale deflections of the trace. These data, with the reset periods excluded, were then sampled as the mean difference between the detector outputs in each cycle of the alternate phase states, producing chopped data. This new dataset was then averaged by angle over the five rotations. Then we removed artifacts; a dc offset, the fundamental of the rotation frequency and the fundamental of the reset frequency. The result is shown in Fig. 3B. We then applied matched filtering to test for the presence of a planet. For an array with the dimensions $60 \mathrm{~m}$ and $10 \mathrm{~m}$ for the long and short axes respectively, nulling across the short baselines, we predict for a planet at $1 \mathrm{AU}$ from its star 10 pc distant, at a wavelength of $10.6 \mu \mathrm{m}$, the "chopped" planet signal shown in Fig. 1C. To measure this putative planet signal we formed a correlation function

$\Phi(R, \theta)=\frac{\sum S * T(R, \theta)}{\sqrt{\sum T(R, \theta) * T(R, \theta)}}$ 
where $S$ is the detected signal and $T$ is the theoretical planet signal, a template produced as discussed by Mennesson \& Mariotti (1997). The templates are calculated for a 6:1 X-array formation (Lay et al. 2007) of telescope apertures. At a given test radius $R$, all values of $\theta$ were tried, where these variables respectively are the angular radius from the star and the orbital phase of the planet. The rms of the function $\Phi$ is the noise level in the signal for a planet at this radius. Figure $3 \mathrm{C}$ shows a plot of $\Phi$ for all values of $\theta$ and for values of $R$ ranging between 0.2 and $1.75 \mu$ radian (equivalent to distances between $0.4 \mathrm{AU}$ and 3.5 AU). For comparison, Fig. 3D shows a model of $\Phi$ at the same scale for a planet three million times fainter than the star, located at $1 \mathrm{AU}$ from the star. We see qualitatively a strong signal compared to Fig. 3C.

Summarizing the results: normalizing the total input starlight to 1 , the mean starlight suppression due to nulling was $6.65 \times$ $10^{-6}$. After chopping, averaging and matched filtering, the rms output is $1.65 \times 10^{-8}$. Thus the combination of nulling, chopping, rotational averaging and matched filtering suppresses the starlight by a factor of $\approx 60$ million, measured as rms noise divided by starlight input. For comparison, a planet three million times fainter than the star and at a distance of $1 \mathrm{AU}$, will have a signal rms of $1.66 \times 10^{-7}$, which is a factor of 10 above the noise. Putting these numbers into the terms of Fig. 2, with a null about $50 \%$ better than $10^{-5}$ the starlight suppression was about three times better than the test goal. Had the null been exactly at the intended $10^{-5}$ level, (difficult to achieve precisely), the starlight suppression would have been two times better than the test goal.

\section{Conclusions and discussion}

The testbed demonstrated starlight suppression of more than seven orders of magnitude using interferometric nulling. The performance was equivalent to that required to detect a Neptunesized object at a distance of $1 \mathrm{AU}$ from a sun-like star at a distance of $10 \mathrm{pc}$, with a signal to noise ratio of 10 . Such an observation would be achievable with a $6: 1 \mathrm{X}$-array formation with baselines of $10 \mathrm{~m}$ and $60 \mathrm{~m}$ between apertures.

The testbed is also capable of producing realistic faint exoplanet signals for detection by the same procedure. These measurements will be reported in a subsequent paper intended for this journal. The next logical step in mid-infrared starlight suppression development would be to employ spectral fitting, which affords a further level of noise suppression by enabling the detection and compensation of the instability noise. Since this noise is produced by a $1 / \mathrm{f}$ process, it is only weakly reduced by an averaging process. However, instability noise has a spectral signature, which allows it to be corrected for in each detection epoch. Conversion of the PDT to operation with a broadband light source, enabling tests of starlight suppression using spectral fitting, is now being carried out. In the longer term, midinfrared nulling interferometry also needs to be tested at realistic fluxes, which will require operation at cryogenic temperatures. This work shows that starlight suppression methods proposed for mid-infrared space interferometers are feasible and effective, and one additional step, the demonstration of spectral fitting, will complete the starlight suppression developments required for spectrally characterizing earth-like planets orbiting nearby stars.

Acknowledgements. Kurt Liewer assisted with testbed development and operation. Frank Loya developed the software control system and several electronics systems. Oliver Lay assisted with data analysis techniques and traceability to flight. The research described in this letter was carried out at the Jet Propulsion Laboratory, California Institute of Technology, under a contract with the National Aeronautics and Space Administration.

\section{References}

Booth, A. J., Martin, S. R., \& Loya, F. 2008, in Optical and Infrared Interferometry, Proc. SPIE 7013, 701320-11

Borucki, W., Koch, D., \& Brown, T., et al. 2010, ApJL, submitted [arXiv: 1001.0604]

Bracewell, R. N. 1978, Nature, 274, 780

Charbonneau, D., Berta, Z. K., Irwin, J., et al. 2009, Nature, 462, 891

Colavita, M. M., Serabyn, G., Wizinowich, P. L., \& Akeson, R. L. 2006, in Advances in Stellar Interferometry, Proc. SPIE 6268, 626803

Defrere, D., Lay, O., den Hartog, R., \& Absil, O. 2008, in Optical and Infrared Interferometry, Proc. SPIE 7013, 701321

Defrere, D., Absil, O., Den Hartog, R., Hanot, C., \& Stark, C. 2009, A\&A, 509, A9

Des Marais, D. J., Harwit, M. O., Jucks, K. W., et al. 2002, Astrobiology, 2, 153 Lay, O. P. 2004, Appl. Opt., 43, 6100

Lay, O. P. 2006, in Advances in Stellar Interferometry, Proc. SPIE 6268, 62681A

Lay, O. P., Martin, S. R., \& Hunyadi, S. L. 2007, in Techniques and Instrumentation for Detection of Exoplanets III, Proc. SPIE 6693, 66930A

Martin, S. 2005, in Techniques and Instrumentation for Detection of Exoplanets II, Proc. SPIE 5905, 21

Martin, S. R., Scharf, D., Wirz, R., et al. 2007, in Techniques and Instrumentation for Detection of Exoplanets III, Proc. SPIE 6693, 669309

Mennesson, B., \& Mariotti, J. M. 1997, Icarus, 128, 202 